\title{
Small-fragment genomic libraries for the display of putative epitopes from clinically significant pathogens
}

\author{
T. Henics ${ }^{1}$, B. Winkler ${ }^{1}$, U. Pfeifer ${ }^{1}$, S.R. Gill², M. Buschle1, A. von Gabain ${ }^{1}$, and A.L. Meinke
}

BioTechniques 35:196-209 (July 2003)

\begin{abstract}
Taking advantage of whole genome sequences of bacterial pathogens in many thriving diseases with global impact, we developed a comprehensive screening procedure for the identification of putative vaccine candidate antigens. Importantly, this procedure relies on highly representative small-fragment genomic libraries that are expressed to display frame-selected epitope-size peptides on a bacterial cell surface and to interact directly with carefully selected disease-relevant high-titer sera. Here we describe the generation of small-fragment genomic libraries of Gram-positive and Gram-negative clinically significant pathogens, including Staphylococcus aureus and Staphylococcus epidermidis, Streptococcus pyogenes, Streptococcus agalactiae, and Streptoococcus pneumoniae, Enterococcus faecalis, Helicobacter pylori, Chlamydia pneumoniae, the enterotoxigenic Escherichia coli, and Campylobacter jejuni. Large-scale sequencing revealed that the libraries, which provide an average of 20-fold coverage, were random and, as demonstrated with two $\mathrm{S}$. aureus libraries, highly representative. Consistent with the comprehensive nature of this approach is the identification of epitopes that reside in both annotated and putatively novel open reading frames. The use of these libraries therefore allows for the rapid and direct identification of immunogenic epitopes with no apparent bias or difficulty that often associate with conventional expression methods.
\end{abstract}

\section{INTRODUCTION}

Recent achievements to uncover whole genome sequence information of a large number of organisms $(1,2)$ have contributed to the excavation of biological data on a previously unseen scale (3). With the aid of whole genome sequences of major disease-causing microbial pathogens, our laboratory has developed a comprehensive screening technology to possibly identify all antigenic proteins that represent the ideal repertoire to select potential vaccine components for combating disease (4). A key component of this technology was the construction of small-fragment genomic libraries, which allowed frame-selected random genomic fragments to be expressed on the bacterial cell surface as peptides for selection using disease-relevant high-titer sera.

Although genomic libraries comprising $1-2 \mathrm{~kb}$ random fragments have been applied for large-scale sequencing (5), other applications, such as the identification of unique sequences flanking specific abundant repeats, ideally call for small-fragment libraries (a few hundred base pairs or fewer), the generation of which has been hampered by several constraints $(6,7)$. For example, shearing any source of DNA into small fragments often compromises fragment intactness, leading to weak cloning efficiencies $(8,9)$. Furthermore, the smaller the fragment size, the higher the number of clones in the library that is required to provide a sufficient coverage of the genome of interest.

To support our previously described antigen identification technology (4), we generated small-fragment (30-300 bp) genomic libraries from several clinically significant pathogens, including Staphylococcus aureus and Staphylococcus epidermidis, Streptococcus pyogenes, Streptococcus agalactiae, and Streptococcus pneumoniae, Enterococcus faecalis, Helicobacter pylori, Chlamydia pneumoniae, the enterotoxigenic Escherichia coli, and Campylobacter jejuni. Processing these libraries in our novel frame-selection vector system allowed for the expression of naturally occurring frames and their subsequent presentation on the bacterial cell surface as accessible small protein fragments for screening (4).

Using large-scale sequencing and PCR-based techniques, we have characterized these libraries and shown that they are sufficiently uniform in fragment size, random, and highly representative. We have also tested the performance of some of these libraries in a bacterial surface display-based assay or in a modified ribosome display screen for the identification of potential vaccine candidate antigens (Reference 4 and Weichhart et al., manuscript submitted). We conclude that our libraries represent a high-quality reservoir of expressible genomic fragments to feed a variety of screening technologies for the identification of vaccine candidate antigens or other regulatory and therapeutic gene products. Additionally, the earlier demonstration of the existence of not yet annotated open reading frames (ORFs) (10) is clearly in line 
with the limited accuracy of ORF prediction by bioinformatic tools. Our identification of putative epitopes within non-annotated ORFs further supports the validity of this whole genome-based application, as this approach in principle covers all possibly encoded peptide sequences.

\section{MATERIALS AND METHODS}

\section{Bacterial Strains and Preparation of Genomic DNA}

S. aureus (methycillin-resistant COL strain) and $S$. epidermidis (RP62A strain) were grown overnight under continuous shaking in brain heart infusion (BHI) medium at $37^{\circ} \mathrm{C}$, while $S$. pyogenes (M1 strain), S. agalactiae, and $S$. pneumoniae (capsular serotype 4) were propagated overnight by shaking in Todd-Hewitt Broth at $37^{\circ} \mathrm{C}$, except that S. pneumoniae was sensitive to shaking. Sufficient amounts of pellets of the $H$. pylori strain $\mathrm{KTH} / \mathrm{Du}$ (isolate from a patient with duodenal ulcer) were the generous gift of Dr. Lars Engstrand. With some modifications of two previously published protocols $(11,12)$, we were able to reproducibly isolate high-quality genomic DNA from the $S$. aureus COL strain (13), and this method was successfully adapted to other Gram-positive bacteria. Routinely, $500 \mathrm{~mL}$ bacterial culture were harvested in two equal aliquots, centrifuged at $1600 \times g$ for 15 min, and the supernatant was discarded. Bacterial pellets were carefully resuspended in $26 \mathrm{~mL} 0.1 \mathrm{mM}$ Tris- $\mathrm{HCl}, \mathrm{pH}$ 7.6 , and centrifuged again at $1600 \times g$ for $15 \mathrm{~min}$. Pellets were then taken up in $20 \mathrm{~mL} 1 \mathrm{mM}$ Tris-HCl, pH 7.6, $0.1 \mathrm{mM}$ EDTA, and transferred into sterile 50$\mathrm{mL}$ polypropylene tubes. Ten micrograms of heat-treated RNase A and 200 $\mathrm{U}$ RNase T1 were added to each tube and mixed carefully. Lysostaphin (250 $\mu \mathrm{L})(10 \mathrm{mg} / \mathrm{mL}$, freshly prepared in double-distilled water) was then added to the tubes $(500 \mu \mathrm{L}$ were added for $S$. epidermidis), mixed thoroughly, and incubated at $40^{\circ} \mathrm{C}$ for $10 \mathrm{~min}$ in a shaking water bath under continuous agitation. After the addition of $1 \mathrm{~mL} 10 \%$ sodium dodecyl sulfate (SDS), $40 \mu \mathrm{L}$ proteinase $\mathrm{K}(25 \mathrm{mg} / \mathrm{mL})$, and $100 \mu \mathrm{L}$ pronase $(10$ $\mathrm{mg} / \mathrm{mL})$, the tubes were again inverted several times and incubated at $40^{\circ} \mathrm{C}$ for 5 min in a shaking water bath. $\mathrm{NaCl}$ $(3.75 \mathrm{~mL} 5 \mathrm{M})$ and $2.5 \mathrm{~mL}$ cetyltrimethylammonium bromide (CTAB) [10\% $(\mathrm{w} / \mathrm{v}), 4 \%(\mathrm{w} / \mathrm{v}) \mathrm{NaCl}]$ were then added, and the tubes were further incubated at $65^{\circ} \mathrm{C}$ in a shaking water bath for $10 \mathrm{~min}$. The samples were cooled to room temperature and extracted with $1 \times$ volume of phenol:chloroform:isoamyl alcohol (25:24:1) and with $1 \times$ volume of chloroform:isoamyl alcohol (24:1). Aqueous phases were carefully collected and transferred to fresh, sterile $50-\mathrm{mL}$ tubes. To each tube, $1.5 \mathrm{~mL}$ Strataclean ${ }^{\mathrm{TM}}$ Resin (Stratagene, La Jolla, CA, USA) were added, mixed gently, and incubated for $1 \mathrm{~min}$ at room temperature. The samples were centrifuged, and the upper layers containing the DNA were collected into clean $50-\mathrm{mL}$ tubes. DNA was precipitated at room temperature by adding $0.6 \times$ volume of isopropanol, spooled from the solution with a sterile Pasteur pipet, and transferred into tubes containing $80 \%$ ice-cold ethanol. DNA was recovered by centrifuging the precipitates at 10,000-12,000× g, air-drying, and redissolving in double-distilled water. Isolation of genomic DNA from the Gram-negative Helicobacter and Campylobacter strains and enterotoxigenic $E$. coli was done similarly, except that instead of lysostaphin, the cells were lysed by lysozyme. Genomic DNA from $C$. pneumoniae was purchased from the Department of Pathobiology, School of Public Health and Community Medicine, University of Washington.

\section{Generation of Small Genomic DNA Fragments}

Genomic DNA was mechanically sheared into fragments that ranged in size from 100 to $300 \mathrm{bp}$ using a cuphorn sonicator (Bandelin Sonoplus UV 2200 sonicator, equipped with a BB5 cup horn, $10 \mathrm{~s}$ pulses at $100 \%$ power output) or into fragments of 30-100 bp by mild DNase I treatment (Shotgun ${ }^{\circledR}$ Cleavage Kit; Novagen, Madison, WI, USA). We observed that breaking the DNA into fragments of 150-300 bp by sonication yielded a much tighter fragment size distribution as compared to fragmentation by DNase I (data not shown). However, despite extensive ex- posure of the DNA to hydromechanical shearing force, a further decrease in fragment size could not be efficiently and reproducibly achieved. Therefore, unsheared genomic DNA was exposed to mild DNase I treatment to generate fragments of 30-70 bp in size. Digestion was performed for $5 \mathrm{~min}$ at $20^{\circ} \mathrm{C}$ by adding $6 \mathrm{U}$ DNase I to approximately $12 \mu \mathrm{g}$ genomic DNA in $50 \mathrm{mM}$ Tris$\mathrm{HCl}, \mathrm{pH} 7.5$, and $50 \mu \mathrm{g} / \mathrm{mL}$ BSA in a $60-\mu \mathrm{L}$ reaction volume, in the presence of $10 \mathrm{mM} \mathrm{MnCl}_{2}$ to ensure doublestranded cleavage. The reactions were stopped with $2 \mu \mathrm{L} 0.5 \mathrm{M}$ EDTA, and the fragmentation efficiency was evaluated on high-purity $2 \%$ TAE-agarose gels (Cambrex, Rockland, ME, USA). This treatment resulted in the total fragmentation of the genomic DNA into almost 50-70 bp fragments. Fragments were then blunt-ended using T4 DNA Polymerase (Promega, Madison, WI, USA), according to the manufacturer's instructions, in the presence of $100 \mu \mathrm{M}$ each dNTPs twice to ensure the efficient flushing of the ends. Fragments were used immediately in ligation reactions or frozen to $-20^{\circ} \mathrm{C}$ until further use.

\section{Description of Vectors and Cloning}

The vectors pMAL 4.1 and pMAL 4.31 were constructed on a $\mathrm{pEH} 1$ and pASK75 backbone, respectively, $(14,15)$ with kanamycin resistance and possessed the following features. These constructs harbor a $\beta$-lactamase gene, which is out-of-frame. $\beta$-lactamase is preceded by a leader peptide of the $E$. coli outer membrane protein, OmpA, to ensure the efficient targeting of the chimera to the periplasmic space. In addition, in pMAL 4.31, the leader peptide is tagged with a spacer for more efficient transport (16). A SmaI site serves for library insertion, and the $\beta$ lactamase out-of-frame context is designed in a way that a +1 frame shift restores the $\beta$-lactamase ORF so that the $\beta$-lactamase protein is produced with a consequent gain of ampicillin resistance (the -1 and 0 reading frame results in stop codons that are 15 and 33 bp after the NotI site, respectively). The SmaI site is flanked by an upstream FseI site and a downstream NotI site (a single nucleotide separates the SmaI site from both FseI and NotI), which 
were used for the recovery of the selected fragments (also see Reference 4). Genomic DNA fragments were ligated into the SmaI site of either the vector pMAL 4.1 or pMAL 4.31. Recombinant DNA was electroporated into DH10B electrocompetent cells (Invitrogen, Carlsbad, CA, USA), and the transformants were grown overnight at $37^{\circ} \mathrm{C}$ on LB-agar plates supplemented with either kanamycin $(50 \mu \mathrm{g} / \mathrm{mL})$ or kanamycin $(50 \mu \mathrm{g} / \mathrm{mL})$ and ampicillin $(50 \mu \mathrm{g} / \mathrm{mL})$. The kanamycin/kanamycin plus ampicillin $(\mathrm{K}+\mathrm{A})$ ratio allowed for the initial evaluation of the frameselection efficiency. Calculated transformant numbers for $1 \mu \mathrm{g}$ recombinant plasmid DNA were $1-2 \times 10^{8}$ clones on kanamycin plates, whereas this figure was $5 \times 10^{6}$ to $10^{7}$ clones on $\mathrm{K}+\mathrm{A}$ plates. Thus, the cumulative clone number per library provided from 10 to 30 -fold coverage of the genome size. Important to our comparative analysis, we note here that the larger insert $S$. aureus library (LSA-250) displayed only a 2-fold coverage of the genome. The colonies were harvested from $\mathrm{K}+\mathrm{A}$ plates with no further propagation, and plasmid DNA was extracted. While maintaining approximately the same complexity, frame-selected fragments were recovered from pMAL 4.1 or 4.31 by FseI-NotI digestion and cloned into one of the bacterial surface display platform vectors for subsequent epitope-identification screens (4).

\section{Evaluation of the Libraries}

A single colony PCR assay was used to initially determine the presence of fragments and their size distribution in the libraries. Following confirmation of quality using this initial assay, 500-700 clones per library were routinely sequenced at The Institute for Genomic Research (TIGR, Bethesda, MD, USA) or MWG-Biotech AG (Ebersberg, Germany). From the trimmed sequence data, we were able to more precisely determine the fragment size distribution and the junction intactness (i.e., the presence of intact FseI and NotI restriction sites flanking the inserts with the proximal and distal parts of the SmaI insertion site). These parameters were also used to evaluate the frame-selection accuracy. Se- quences matching any of the ORFs versus non-ORFs in the sense or complementary orientations and chimeric sequences were plotted over the respective genome using the Statistica ${ }^{\circledR}$ 6.0 software. For a more comprehensive assessment of the representation of any genomic fragment, we employed a PCR-based assay on FseI or NotI linearized libraries, where one oligonucleotide corresponded to a vector sequence upstream or downstream of the library insertion site, and the respective reverse or forward primers corresponded to randomly selected sequences of known $S$. aureus ORFs or non-S. aureus ORFs. Additionally, 20 entirely random, in silico-selected oligonucleotides that corresponded to 20 mer of the $S$. aureus chromosome were also used in a similar PCR assay to assess the presence of those sequences in both orientations. The PCR products were sequenced and aligned back to the genome sequence to confirm specificity. Table 1 lists the oligonucleotides used in these experiments.

\section{RESULTS}

\section{Fragment Size Distribution and Frame-Selection Efficiency of the Genomic Libraries}

The construction of libraries with acceptable complexity and representation largely relies on the random and reproducible fragmentation of the genomic DNA and highly efficient cloning conditions. The fragmentation method of choice is ideally devoid of sequenceassociated biases. Although sonication or other hydromechanical shearingbased techniques usually yield good results, the generation of increasingly smaller fragments may severely compromise the intactness of the DNA $(7,8,17)$. Enzymatic techniques generally operate with some sequence-dependent bias but can produce fragments with unlimitedly small size under carefully selected conditions $(18,19)$. Importantly, DNase I can be directed toward double-stranded cleavage in the presence of manganese ions, a condition that minimizes the occurrence of sequence-dependent biases (18). Based on these considerations, we employed sonication to obtain genomic DNA fragments of 100-300 bp and DNase I to generate genomic fragments with sizes as small as $30-100 \mathrm{bp}$. Following careful repair and blunt-ending, these fragments were introduced into our novel frame-selection vector, which allowed the elimination of most random sequences that possessed a STOP codon in the expressed reading frame (4). Since recircularized vectors did not promote $\beta$-lactamase expression, we assumed the representation of all ORFderived sequences and sequences of potential but not annotated ORFs that did not possess a STOP codon in their reading frame. After antibiotic selection, 500-1000 randomly picked clones were sequenced and subsequently analyzed. Figure 1 shows the fragment size distribution of 10 different genomic libraries. This analysis revealed that, although there are slight differences in the size distribution between the libraries, this parameter is generally uniform because, in most instances, approximately $80 \%$ of the fragments fall into a defined size range (Figure 1).

Scanning through the sequences of individual clones allowed us to carefully assess the quality of the library insertion sites/junctions and to determine the efficiency of the frame-selection system. Our evaluation revealed that more than $95 \%$ of the fragments possessed the correct length that allowed the readthrough of the $\beta$-lactamase gene (4). Thus, the elimination of clones that contained fragments with incorrect frames and those with recircularized vectors was in accordance with the average of 20 -fold reduction of clones on the $\mathrm{K}+\mathrm{A}$ plates. Due to the construction principle of our frame-selection vector system, these fragments could, in principle, be in any of the six possible frames in relation to the naturally occurring ORF. Therefore, with precise localization of the fragments on their corresponding ORFs, the actual reading frames were determined, and the enrichment of naturally occurring fragments (i.e., fragments that aligned to the natural frame of the annotated sequence) was evaluated. Our results showed that, when compared to the theoretically calculated approximately $15 \%$ representation of naturally occurring ORF sequences in a random pool of fragments (six possible 
Table 1. Oligonucleotide Primers Used to Evaluate Fragment Representation

\begin{tabular}{|c|c|c|}
\hline ORF & Forward Primer & Reverse Primer \\
\hline$S A 1610$ & 5'-TCAGCAGCAACGACTCAAGCAAATTCAG-3' & 5'-GGAGGTGCATAATGTATTCCAGGTCTG-3' \\
\hline$S A 2613$ & 5'-ACTAAAGTTGACAAAGTACCAACGCTTAAAGC-3' & 5'-CTTCTAAAACGATAAATACATCGATATTATCGTAAGG-3' \\
\hline SA2006 & 5'-ACGTGGATCCAATTCAGCTCATAAAGACTCTCAA-3' & 5'-TGCAGCGGCCGCTTATCCTTCTTTATAAGGTTTATTGTC-3' \\
\hline SA2019 & 5'-ACGTGAATTCAAGGATAACTTAAATGGAGAAAAACCA-3' & 5'-TGCAGCGGCCGCTTATCGTCGCTGTGATTCGTTTTTATTTTTC-3' \\
\hline SA1781 & 5'-ACGTGGATCCGCAGAAAATACAAATACTTCAGATAA-3' & 5'-TGCAGCGGCCGCTTAAGTTTTTGGTAGCATTTTAGATTGACTAAG-3' \\
\hline SA2584 & 5'-ACGTGGATCCGCTGAAGTAAACGTTGATCAAGCA-3' & 5'-TGCAGCGGCCGCTTAGAATCCCCAAGCACCTAAACCTTG-3' \\
\hline$S A 1231$ & 5'-ACGTGGATCCCTAGAGGCACAATTTTTTACTGATAC-3' & 5'-TGCAGCGGCCGCTTAGAATCCCCAAGCACCTAAACCTTG-3' \\
\hline SA723 & 5'-ACGTGGATCCCAAGCTTCTACACAACATACAGTA-3' & 5'-TGCAGCGGCCGCTTAATGGATGAATGCATAGCTAGAAAC-3' \\
\hline SA2291 & 5'-ACGTGGATCCTCTGAGCAAGATAACTACGGTTATAATC-3' & 5'-TGCAGCGGCCGCTTAGTGAATGAAGTTATAACCAGCAGC-3' \\
\hline SA2511 & 5'-CGCTGGCCGGCCGGCCAAGGTGGCCAAAATAGC-3' & 5'-CGCGTTGCGGCCGCGGCCGCTTACTTTTGGAAG-3' \\
\hline SA1231 & 5'-CTGATACTGGACAACATAGAGA-3' & 5'-AAGTAACGTTATCTTTCGAATGGT-3' \\
\hline hHsp70 & 5'-GAGAGTGACTCCCGTTGTCC-3' & 5'-TCTGCATGTAGAAACCGGAA-3' \\
\hline ORF & Reverse Primer & Reverse Primer \\
\hline SA1092 & 5'-GCTAATGTGTATTGAATTAA-3' & 5'-GAATATCATCCATATTTAAC-3' \\
\hline SA1835 & 5'-TTATGAAGATTATTGAAAAGA-3' & 5'-AAATCGAAGAGCATGTCGAC-3' \\
\hline non-ORF & 5'-TTCCATTAATTTACTACCAC-3' & 5'-TGCGCCACGATGATGTCTGA-3' \\
\hline$S A 1534$ & 5'-TGCAGTTGCTACAATTACAC-3' & 5'-TCAAGCTGAAGCAGTTATGG-3' \\
\hline SA0944 & 5'-ATCTACAATCATACTCATGT-3' & 5'-GGTACAACAATTGTTGTGAT-3' \\
\hline SA0260 & 5'-ATATAGTACGGCAGGTTTGC-3' & 5'-ATAACTTTTGGTGTCGTGTG-3' \\
\hline$S A 0572$ & 5'-GACTTAGCATATCCAGGTGT-3' & 5'-AGTTGCATAGGCATTATGTT-3' \\
\hline SA2738 & 5'-GATTCAGACAGTGATTCAGA-3' & 5'-GTCACTGCAAGCACCATTGA-3' \\
\hline SA0979 & 5'-GATTTAGAATTGTTGATTGC-3' & 5'-GTATTCATACCTTGAACACC-3' \\
\hline SA0828 & 5'-CACCGTCACATATTGAGTGA-3' & 5'-TCTTCAAATTGTTCCTCTGA-3' \\
\hline pMAL 4.1 & 5'-GCACTGGCTGGTTTCGCTACCG-3' & ard) \\
\hline pMAL 4.1 & 5'-TCACCAGCGTTTCTGGGTGGCGGCCGC-3' & rse for Figure 3) \\
\hline pMAL 4.1 & 5'-TTACCAATGCTTAATCAGTGA-3' & rse for Figure 5) \\
\hline
\end{tabular}

frames and an arbitrary $10 \%$ of noncoding genome sequence) in our small (30-100 bp) and large (100-300 bp) fragment libraries, these numbers were approximately $32 \%$ and $66 \%$, indicating a respective 2- and 4-fold enrichment of naturally occurring sequences. As expected, this number was always about 2-fold higher in the larger fragment libraries because of the higher probability of encountering a STOP codon in the "wrong" frames.

\section{The Genomic Libraries are Random}

With the bulk sequence information of the randomly picked clones on hand, we then determined how these se- quences aligned to the chromosome of the pathogen, a simple measure of distribution randomness of sampled genomic fragments. Trimmed clone sets of each library were aligned against the corresponding whole genome sequence using the Basic Local Alignment Search Tool (BLAST) at an $e$-value of $10^{-3}(20)$. The clone sequences were aligned separately against all annotated ORFs and against non-coding (contig) sets of the corresponding genomes, and the number of sequences with clear hits was determined (Figure 2B). As expected, chimera formation as a result of our blunt-ended cloning strategy was also revealed, and the precise number of chimeric sequences was determined.
Based on data obtained from 25 libraries, the average appearance of chimeric fragments in small (30-100 bp) and larger (100-300 bp) fragment libraries was $14 \%$ and $2.5 \%$, respectively. Similar to the results with $S$. aureus (4), the graphic alignment of the genomic fragment sequences of libraries for four additional organisms ( $S$. pyogenes, $S$. pneumoniae, H. pylori, and C. jejuni) demonstrated that the fragments distributed over the corresponding bacterial chromosomes in a highly random manner (Figure 2A). Distinct plotting of ORF versus non-ORF hits demonstrated a higher ORF hit ratio, as expected from the high proportionality of coding sequences in bacterial genomes. 


\section{The Genomic Libraries are Complex and Representative}

The random distribution of the fragments over the respective genomes and the calculated complexity (an average of 20 -fold coverage of the corresponding genome) are not per se sufficient parameters to evaluate the representative nature of the libraries (i.e., to assess the presence of any randomly selected genomic sequence in the library). To address this question, we tested the LSA-50 S. aureus library using a PCR-based approach. We employed primers annealing at known positions upstream and downstream of the SmaI library insertion site of the pMAL 4.1 frame-selection vector (Figure 3A) and both forward and reverse primers for a panel of 14 ORFs in the $S$. aureus genome (Figure 3B). Thus, the PCRs marked the presence or absence of the targeted genomic region in duplicates and in a complimentary fashion. Primers employed in reactions 1 and 20,15 and 16, and 23 and 24 identify different regions within ORFs SA2291 and SA1231, respectively. Note that regions that are distinct from those marked by the ORF-specific primers used in this study have previously been identified for several ORFs in our novel bacterial surface display-based screen using the very same library. These include SA2006, SA2019, SA2367, SA1781, SA2584, SA0723, and SA2291. Additionally, regions of SA2291 and SA2367 were identified with high and low frequency in the screen, respectively. Primers that were specific for other ORFs that were not identified in the screen including SA1610, SA2613, SA1231, and SA2511 and for ORFs of non-S. aureus origin were also included.
The upper panel in Figure 3B presents a representative set of PCRs, in which the LSA-50 S. aureus genomic library was screened for the presence of fragments derived from the indicated ORFs. The evaluation of the corre- sponding complementary reactions for each ORF sequence (i.e., when both ORF-specific complementary primers were analyzed with both vector primers) allowed us to conclude that all selected genomic sequences were pre-

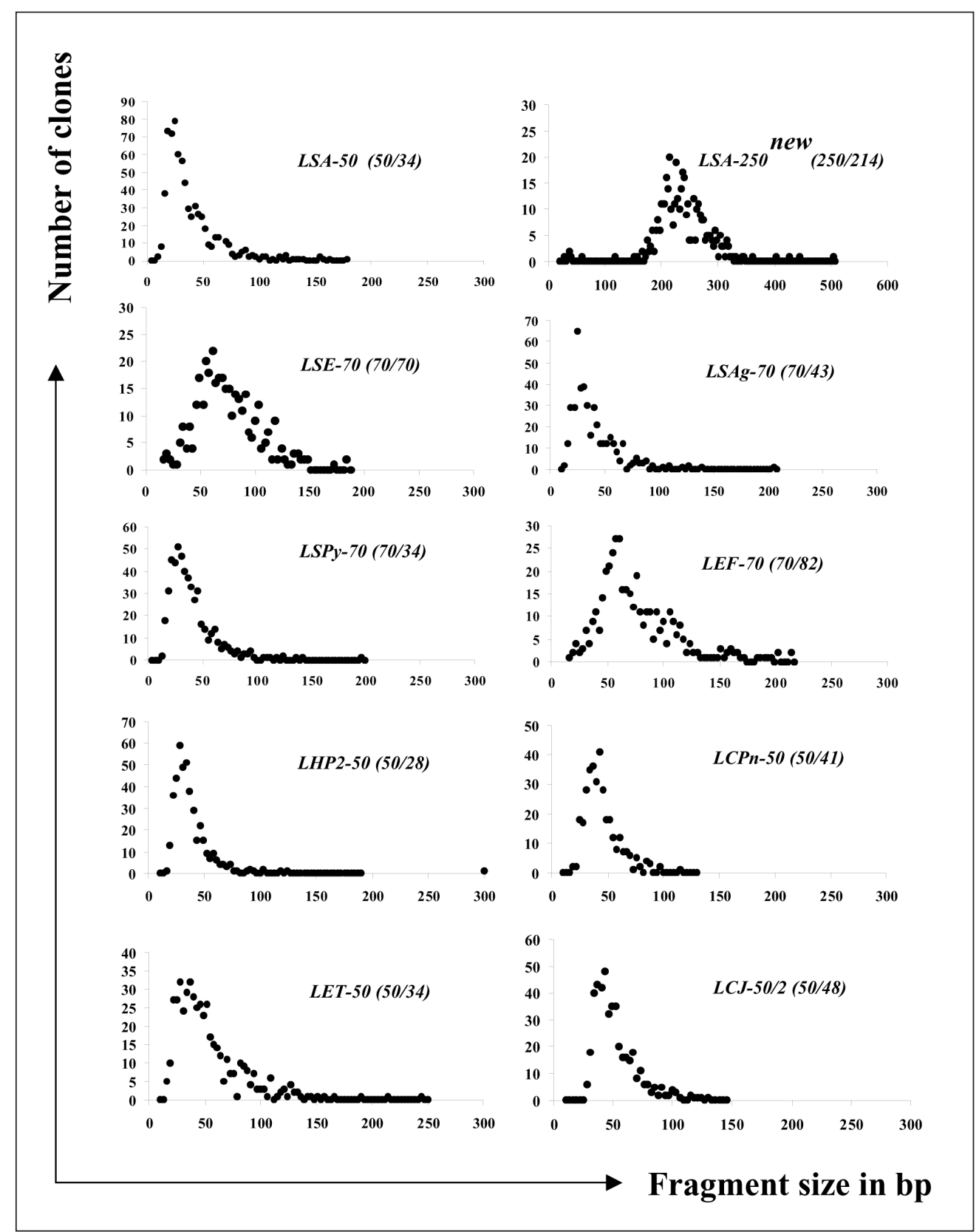

Figure 1. Fragment size distribution of 10 different small-fragment genomic libraries. Libraries were constructed to possess 30-70 bp long genomic fragments, except for LSA-250 new, which was constructed with larger fragments (approximately $300 \mathrm{bp}$ ). Genomic DNA fragments were end-repaired and size-separated for cloning into vectors pMAL 4.1 or 4.31. Approximately 600 clones were sequenced, and the precise length of these fragments was determined. The numbers of clones with various sizes (in agreement with the $3 n+1$ rule of frame selection) were plotted for each library. The names of the libraries are indicated within the graphs: LSA-50 and LSA-250 new, S. aureus; LSE-70, S. epidermidis; LSAg-70, S. agalactiae; LSPy-70, S. pyogenes; LEF-70, E. faecalis; LHP2-50, H. pylori; LCPn-50, C. pneumoniae; LET-50, enterotoxigenic E. coli; LCJ-50, C. jejuni. In bracelets, the number of intended fragment size versus the determined average fragment size is given in base pairs. 
sent in both orientations in the library. As expected, the size of these products varied due to the variable size of the fragments and agreed in both sets of reactions (Figure 3B, upper left and right panel) with the situation of the vector primers, relative to the library insertion site of the construct (Figure 3A). Similar results were obtained with another $S$. aureus library, LSA-250, the average fragment size of which was larger (about $250 \mathrm{bp}$ ) (Figure 3B, lower panels). However, some reactions did not yield a clearly identifiable product (e.g., lanes $3,4,6$, and 7 on the lower-left panel or lanes $6,9,23$, and 2 on the lower-right panel). We assumed that some of these reactions were indeed negative and attributable to the lack of the fragments in the library because the complexity of LSA-250 corresponded to

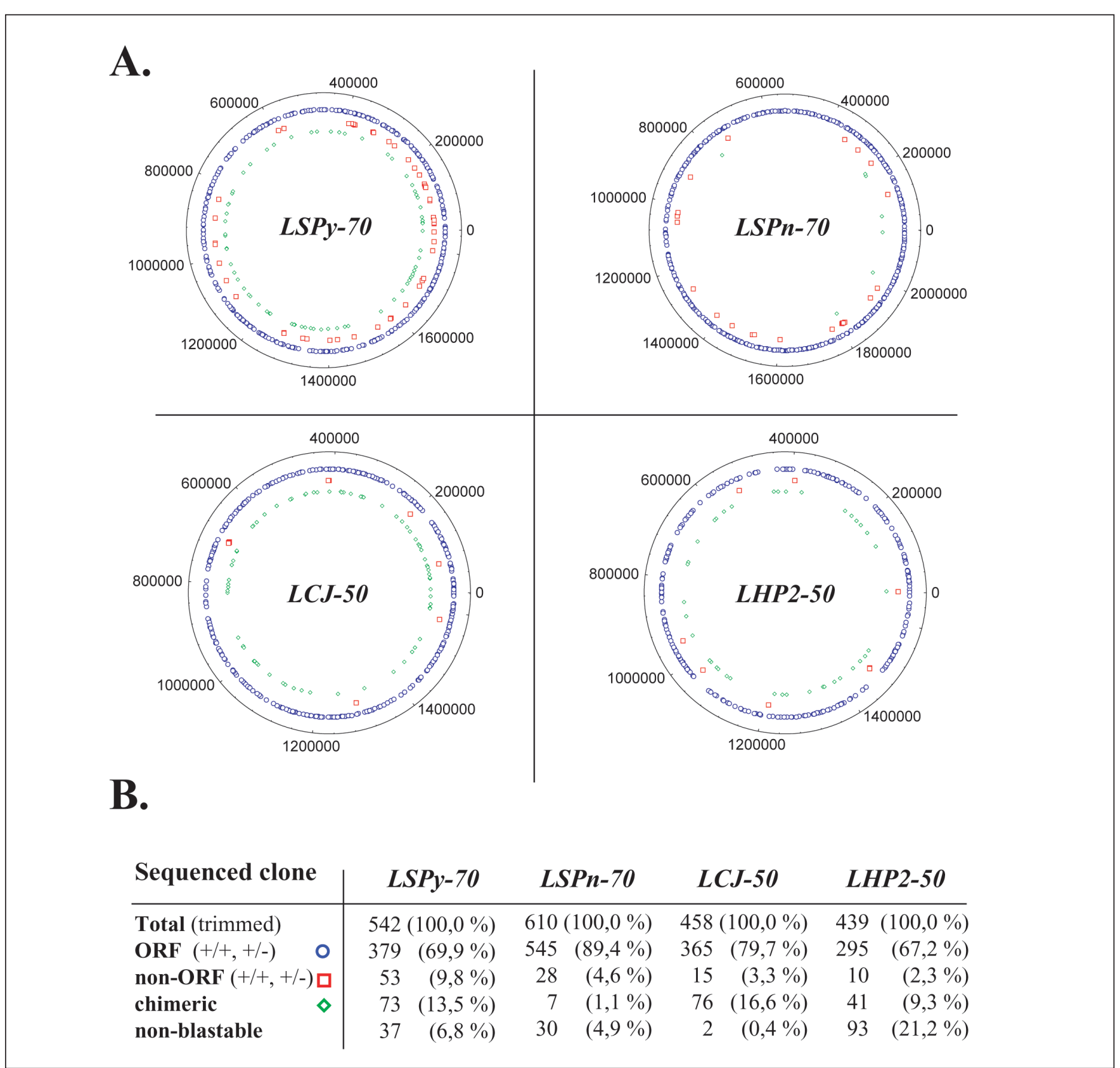

Figure 2. Graphic illustration of the distribution of randomly sequenced clones from small-fragment genomic libraries of $S$. pyogenes (LSPy-70), $S$. pneumoniae (LSPn-70), C. jejuni (LCJ-50), and H. pylori (LHP2-50) over their respective chromosomes. (A) Clones (576) were sequenced for each library, except that this figure was 672 for LSPn-70. Raw sequences were trimmed to eliminate vector sequences, and successfully trimmed sequences were aligned to the fully annotated whole genomes using BLASTN. The sequences were blasted separately against open reading frame (ORF) and non-ORF (contig) database sets to ensure clarity. Blue circles indicate fully matching sequences to annotated ORFs in $+/+$ or $+/$ - orientations. Red rectangles represent fully matched clones to non-coding chromosomal sequences in $+/+$ and $+/$ - orientations. Green diamonds position chimeric clones where clear start codon could be defined within the sequence for chromosomal positioning. Numeric distances in base pairs are indicated over each circular genome for orientation. (B) Partitioning of various clone sets within each library is given in numbers and percentage. 
only about 2-fold coverage of the genome. For this reason, we constructed another larger fragment library of $S$. aureus (LSA-250 ${ }^{\text {new }), ~ t h e ~ f r a g m e n t ~ s i z e ~}$ distribution of which is shown in Figure 1. A set of control reactions was also performed to evaluate any background noise in these experiments and to ensure specificity. Figure 4 show that no obvious products were apparent when the linearized libraries served as templates in PCRs using the pMAL 4.1 vectorspecific primers that annealed upstream (lanes 1 and 2) or downstream (lanes 3 and 4) of the library insertion junction. In contrast to the positive control, which yielded the expected products (lanes 7 and 8), no PCR products were obtained when the eukaryotic ORF [human inducible $70-\mathrm{kDa}$ heat-shock protein (hHsp70)]-specific primers were tested together with the corresponding vectorspecific primers (lanes 5 and 6).

To further test the representative value of the two $S$. aureus libraries, similar PCR analysis was performed with a set of primers that were randomly selected in silico from the genome of this organism (Table 1), except that the reverse vector primer in this experiment was located further downstream of the library insertion site, giving rise to a 801-bp product from an empty vector when used together with the upstream vector primer (Figure 5A). Figure 5B lists the ORFs that corresponded to all randomly selected primers and indicates that three of the 20 primer sequences were not within annotated ORFs. Figure 5A demonstrates that all 40 reactions were positive with LSA-50 in both orientations, indicating high representation for this library. Given that approximately $90 \%$ of a prokaryotic chromosome comprises coding information, the fact that our libraries also contained non-ORF fragments, further confirmed their highly random and representative nature. When similar PCR analysis was performed using LSA-250, we found that, despite multiple attempts and conditions, only 35 of 40 reactions were positive (Figure 5A). Importantly, this result was in agreement with that obtained with the set of primers shown in Figure $3 \mathrm{~B}$ and underscores the importance of sufficient genome coverage to represent any given fragment in these libraries. Finally, 13 (LSA-250) and 8 (LSA-50)
A.

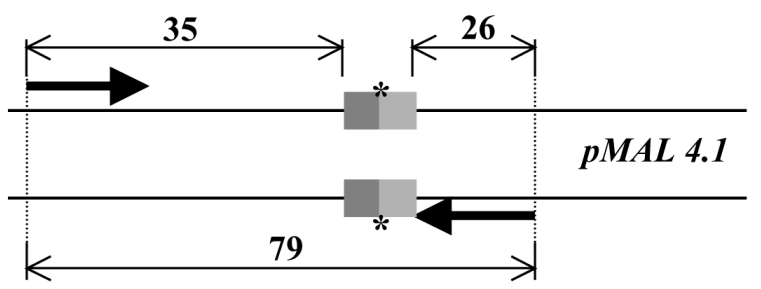

B.

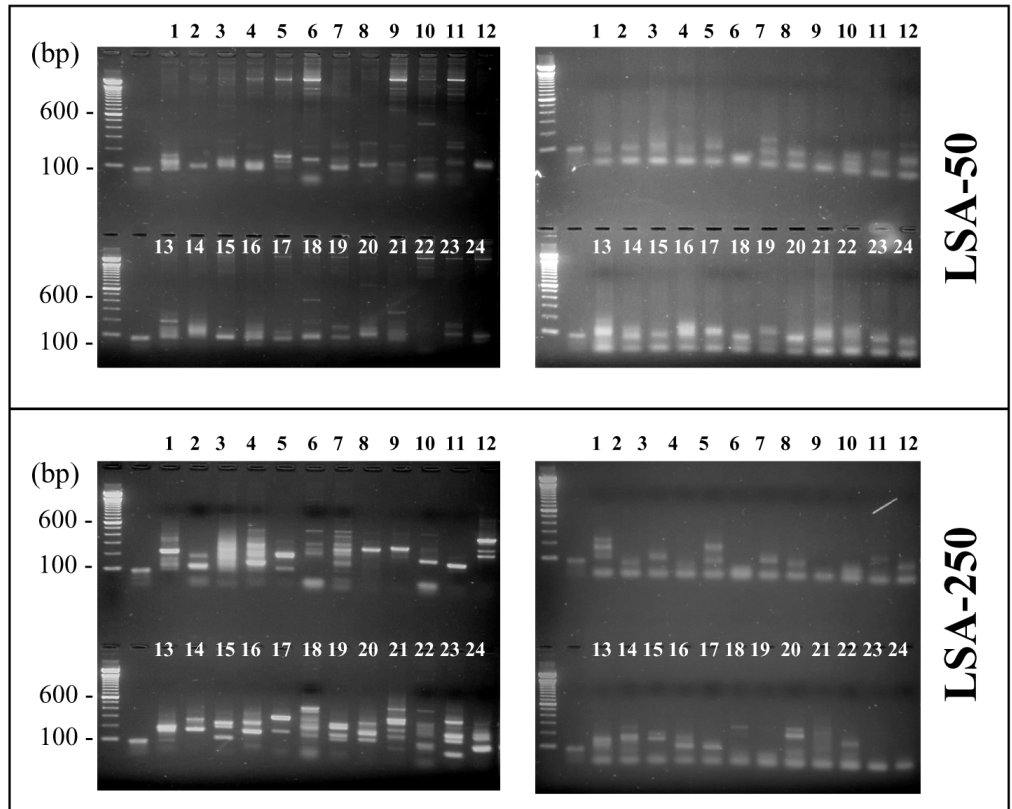

\begin{tabular}{c|c|c||c|c|c} 
Lane & Primer Orientation & ORF & Lane & Primer Orientation & ORF \\
\hline $\mathbf{1}$ & reverse & SA2291 & $\mathbf{1 3}$ & forward & SA2584 \\
\hline $\mathbf{2}$ & reverse & SA2367 & $\mathbf{1 4}$ & reverse & SA2584 \\
\hline $\mathbf{3}$ & forward & SA1610 & $\mathbf{1 5}$ & forward & SA1231 \\
\hline $\mathbf{4}$ & reverse & SA1610 & $\mathbf{1 6}$ & reverse & SA1231 \\
\hline $\mathbf{5}$ & forward & SA2613 & $\mathbf{1 7}$ & forward & SA0723 \\
\hline $\mathbf{6}$ & reverse & $\mathbf{S A 2 6 1 3}$ & $\mathbf{1 8}$ & reverse & SA0723 \\
\hline $\mathbf{7}$ & forward & $\mathbf{S A 2 0 0 6}$ & $\mathbf{1 9}$ & forward & SA2291 \\
\hline $\mathbf{8}$ & reverse & SA2006 & $\mathbf{2 0}$ & reverse & SA2291 \\
\hline $\mathbf{9}$ & forward & SA2019 & $\mathbf{2 1}$ & forward & SA2511 \\
\hline $\mathbf{1 0}$ & reverse & SA2019 & $\mathbf{2 2}$ & reverse & SA2511 \\
\hline $\mathbf{1 1}$ & forward & SA1781 & $\mathbf{2 3}$ & forward & SA1231 \\
\hline $\mathbf{1 2}$ & reverse & SA1781 & $\mathbf{2 4}$ & reverse & SA1231
\end{tabular}

Figure 3. Evaluation of the representative nature of $S$. aureus libraries using known genomic sequences. (A) Schematic illustration of the library-cloning region of the pMAL 4.1 frame-selection vector system. The SmaI library insertion site is indicated with an asterisk, and flanking FseI and NotI sites are colored in dark and light gray, respectively. The positions of the two primers used in the PCR analyses are indicated (arrows), and the distances of the furthest nucleotides of the primer attachment sites from the first nucleotides of the FseI and NotI recognition sites are given in nucleotides. The PCR product yielded by the two primers from an empty vector template is $79 \mathrm{bp}$ in length. Table 1 gives the sequences of the vector primers. (B) PCR analysis that shows the representative nature of the $S$. aureus genomic libraries, LSA-50 and LSA-250. Left and right panels show the results obtained using open reading frame (ORF)-specific primers, together with vector primers upstream or downstream, respectively, of the library insertion site. The orientation and specific numbering are indicated in the lower panel of the figure. The unlabeled lanes in each panel correspond to the empty vector control where the two vector primers were used in the PCR. 
PCR products obtained with the random primers were sequenced and all found to be identical with fragments that contained the original primer sequence of the corresponding genomic regions (data not shown). These results demonstrate that the random PCR screening is a quick and efficient tool for the assessment of the presence or absence of any given fragment in the library.

\section{DISCUSSION}

The recent acceleration of drug and vaccine discovery generated by the "genomic revolution" of molecular biology largely relies on bulk-scale screening technologies that require high-complexity libraries. Access to the full genome sequence of a growing number of organisms makes genomic library-based approaches very attractive. The success of constructing such libraries assumes good-quality genomic DNA in sufficient quantity, highcloning efficiency, and access to largescale sequencing $(21,22)$.

In this study, randomly fragmented

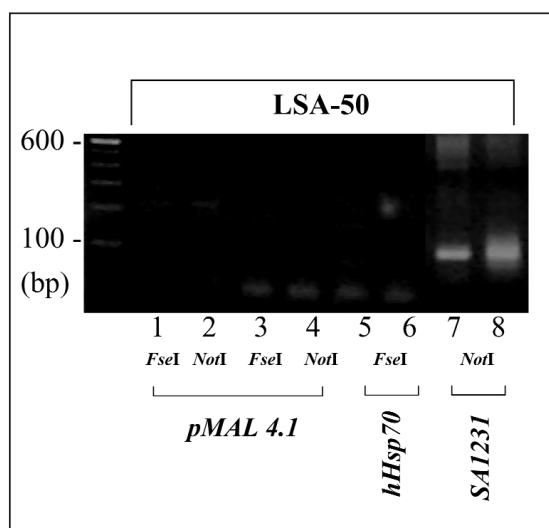

Figure 4. Control PCR analysis of the $S$. aureus small-fragment genomic library, LSA-50. Both forward and reverse primers specific for the eukaryotic open reading frame (ORF); human 70$\mathrm{kDa}$ heat-shock protein (hHsp70) as negative control (lanes 5 and 6); and the $S$. aureus ORF, SA1231, as positive control (lanes 7 and 8) were used. These two ORFs were targeted using FseI and Not $\mathrm{I}$ linearized libraries (indicated at the bottom of the gel), and the corresponding downstream and upstream pMAL 4.1 vector-specific primers, respectively, as explained in Figure 3. The same library, linearized with the indicated enzymes, served as a template in PCRs where only the upstream (lanes 1 and 2) and downstream (lanes 3 and 4) vector primers were used. DNA size markers are shown on the left side of the figure. genomic DNA fragments of human bacterial pathogens were cloned into our novel $\beta$-lactamase-based frame-selection vector system to facilitate the enrichment of naturally occurring sequences in the libraries (detailed de- scription of the vector is given in Reference 4). With the aid of large-scale sequencing of approximately 600 randomly selected clones per library, we were able to determine the precise length of each fragment and their distri-

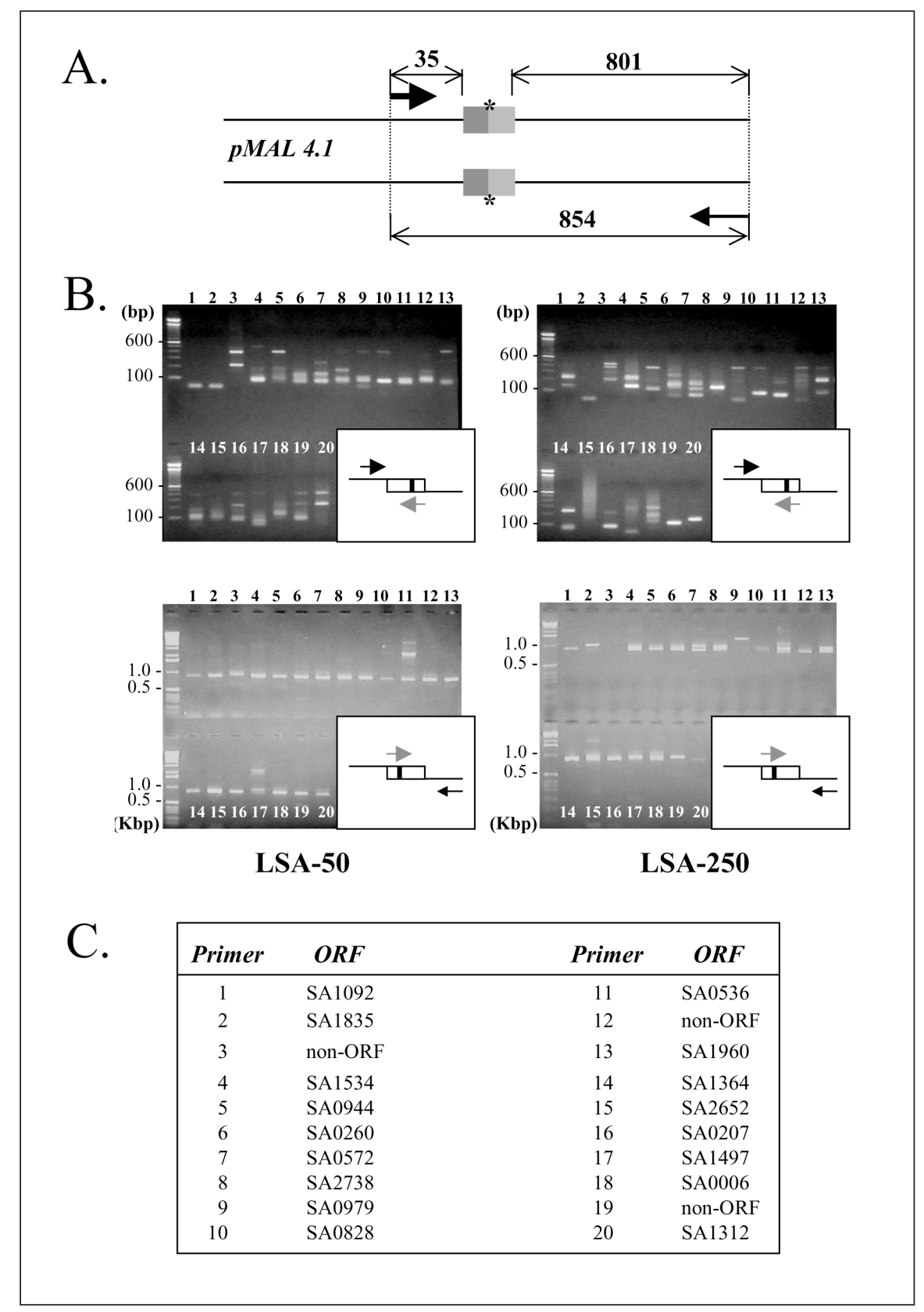

Figure 5. Evaluation of the representative nature of $\boldsymbol{S}$. aureus libraries using random genomic sequences. (A) Schematic illustration of the library-cloning region of the pMAL 4.1 frame-selection vector system as described for Figure 3A, except that distances in base pairs are shown with the downstream-most vector primer. (B) PCR analysis of the $S$. aureus small-fragment genomic libraries, LSA-50 and LSA-250, using primers (gray arrow) corresponding to 20 randomly selected 20 -mer sequences of the $S$. aureus genome, together with both a forward $(\rightarrow)$ and a reverse $(\leftarrow)$ primer annealing to regions upstream or downstream of the library-cloning site of pMAL 4.1, as indicated in panel A. Left and right panels show the results obtained using LSA-50 and LSA-250, respectively. The numbering of genomic regions (17 ORFs and three non-ORF regions) for which the randomly selected primers were specific is listed in panel C. 
bution over the physical map of the chromosomes of $S$. aureus, $S$. pyogenes, and $S$. pneumoniae, $H$. pylori, and C. jejuni. The alignment of the trimmed sequences demonstrated that their distribution throughout the genomes was essentially random, which suggested that the fragmentation and subsequent frame selection did not introduce any obvious bias to the libraries (Figure 2). Moreover, this also allowed us to analyze the intactness of cloning junctions for each individual sequenced clone and the performance of the frame-selection system. Evaluating the fragment lengths revealed that over $95 \%$ of the clones with intact cloning junctions followed what we call the " $3 n+1$ rule"; that is, only those fragments directed the expression of the downstream $\beta$-lactamase gene that possessed the +1 frame out of the three possibilities as a consequence of random fragmentation (8). We attribute the appearance of the majority of clones with corresponding fragment lengths of " $3 n-1$ " or " $3 n$ " to sequencing errors because such clones enrich among those with longer fragments; that is, where the sequence reading accuracy is lower (200-300 bp downstream of the primer attachment site). In agreement with this, lowering the stringency of BLASTN, several of such sequences could be assigned to the genome. Additionally, manually checking several of these sequences identified the sequencing error (data not shown). Importantly, our extended analysis revealed that, in correlation with the fragment length, an increasing proportion of the fragments corresponded to naturally occurring frames of their respective ORFs. This demonstrates the capacity of our libraries to considerably enrich for naturally occurring genomic sequences relative to the theoretically calculated representation of such sequences in a nonselected random pool. Additionally, a powerful feature of our libraries selected in such a manner is that the subsequent recovery of these fragments via the flanking FseI and NotI sites allows for the direct in-frame transfer of the libraries into any platform vector used in further screening/ assay systems, such as bacterial surface display (4) or phage display.

We performed an additional complex analysis to assess the representative na- ture of the libraries. These experiments involved the PCR-based detection of known or entirely random sequences of chromosomal origin and confirmation of these results by the sequencing of PCR products. The successful detection and verification of these sequences in the case of our "prototype" $S$. aureus libraries revealed that the libraries were highly representative (Figures 3 and 5). The observed lack of some randomly selected sequences in the low coverage LSA-250 argues that the complexity of genome coverage cannot be compromised without losing representation.

We have also demonstrated that, using LSA-50 and LSA-250, it was possible to identify more than 100 antigenic proteins in bacterial surface display- or ribosome display-based screens (Reference 4 and Weichhart et al., manuscript submitted). These techniques require pathogen-related, disease-specific highantibody titer human sera for powerful target selection. However, the success of relevant antigen identification can be hampered if the complexity and representative nature of the library is compromised. Although the fragment size distribution in the libraries was somewhat variable in its reproduction, a property that we partly attribute to the diverse fragmentation response of the genomic DNA preparations, we found that this did not interfere with the subsequent presentation of the fragments in the applied display technologies. We also did not observe any bias in the $\beta$-lactamasebased frame-selection process due to the occasional heterogeneity of fragment size in the libraries (Figure 1A). Therefore, we are confident that our genomic libraries are of great value, and they provide a comprehensive and direct representation of putative epitopes of any potential antigen in high-throughput screening or identification techniques to define synthetic vaccine components. In conclusion, we described a reproducible and widely applicable method of genomic library construction of human pathogenic bacteria, in which a complex and representative pool of proper ORFselected, random genomic fragments are expressed for a variety of subsequent high-throughput screening techniques to identify, for example, antigenic entities of potential interest for new-generation vaccine development.

\section{ACKNOWLEDGMENTS}

We are grateful to Bernd Mayer for the graphic illustrations of clone sequence distribution over the bacterial chromosomes and Barbara Ginda for bioinformatic support. We thank Max Birnstiel for his stimulating ideas and support throughout this project.

\section{REFERENCES}

1.Fraser, C.M. and R.D. Fleischmann. 1997. Strategies for whole microbial genome sequencing and analysis. Electrophoresis 18:1207-1216

2.Nelson, K.E., I.T. Paulsen, J.F. Heilderberg, and C.M. Fraser. 2000. Status of genome projects for nonpathogenic bacteria and archaea. Nat. Biotechnol. 18:1049-1054.

3.Palsson, B. 2000. The challenges of in silico biology. Nat. Biotechnol. 18:1147-1150.

4.Etz, H., D. Bui Minh, T. Henics, A. Dryla, B. Winkler, C. Triska, A.P. Boyd, J. Söllner, et al. 2002. Identification of in vivo expressed vaccine candidate antigenes from Staphylococcus aureus. Proc. Natl. Acad. Sci. USA 99:6573-6578.

5.Andersson, B., M.A. Wetland, J.Y. Ricafrente, W. Liu, and R.A. Gibbs. 1996. A "double adaptor" method for improved shotgun library construction. Anal. Biochem. 236:107-113

6.Karagyozov, L., I.D. Kalcheva, and V.M. Chapman. 1993. Construction of random small-inset genomic libraries highly enriched for simple sequence repeats. Nucleic Acids Res. 21:3911-3912.

7.Oefner, P.J., S.P. Hunicke-Smith, L. Chiang, F. Dietrich, J. Mulligan, and R.W. Davis. 1996. Efficient random subcloning of DNA sheared in a recirculating point-sink flow system. Nucleic Acids Res. 24:3879-3886.

8.Bankier, A.T. 1993. Generation of random fragments by sonication. Methods Mol. Biol. 23:47-50.

9.Hengen, P.N. 1997. Shearing DNA for genomic library construction. Trends Biochem. Sci. 22:273-274.

10.Jungblut, P.R., E.-C. Müller, J. Mattow, and S.H.E. Kaufmann. 2001. Proteomics reveals open reading frames in Mycobacterium tuberculosis H37Rv not predicted by genomics. Infect. Immun. 69:5905-5907.

11.Betley, M.J., S. Lofdahl, B.N. Kreiswirth, M.S. Bergdoll, and R.P. Novick. 1984 , Staphylococcal enterotoxin A gene is associated with a variable genetic element. Proc. Natl. Acad. Sci. USA 81:5179-5183.

12.Sohail, M. 1998. A simple and rapid method for preparing genomic DNA from Gram-positive bacteria. Mol. Biotechnol. 10:191-193.

13.Archer, G.L. and D.M. Niemeyer. 1994. Origin and evolution of DNA associated with resistance to methicillin in staphylococci. Trends Microbiol. 2:343-347.

14.Hashemzadeh-Bonehi, L., F. MehraeinGhomi, C. Mitsopoulos, J.P. Jacob, E.S. 
Hennessey, and J.K. Broome-Smith. 1998 Importance of using lac rather than ara promoter vectors for modulating the levels of toxic gene products in Escherichia coli. Mol. Microbiol. 30:676-678.

15.Skerra, A. 1994. Use of the tetracycline promoter for the tightly regulated production of a murine antibody fragment in Escherichia coli. Gene 151:31-135.

16.Kajava, A.V., Zolov, S.N., Kalinin, A.E., and M.A. Nesmeyanova. 2000. The net charge of the first 18 residues of the mature sequence affects protein translocation across the cytoplasmic membrane of Gram-negative bacteria. J. Bacteriol. 182:2163-2169.

17.McKee, J.R., C.L. Christman, W.D. O'Brien, and S.Y. Wang. 1977. Effects of ultrasound on nucleic acid bases. Biochemistry 16:4651-4654.

18.Anderson, S. 1981. Shotgun DNA sequencing using cloned DNase I-generated fragments. Nucleic Acids Res. 9:3015-3027.

19.Fitzgerald, M.C., P. Skowron, J.L. Van Etten, L.M. Smith, and D.A. Mead. 1992. Rapid shotgun cloning utilizing the two base recognition endonuclease CviJI. Nucleic Acids Res. 20:3753-3762.
20.Altschul, S.F., W. Gish, W. Miller, E.W. Myers, and D.J. Lipman. 1990. Basic local alignment tool. J. Mol. Biol. 215:403-410.

21.Syn, C.K.C. and S. Swarup. 1999. A scalable protocol for the isolation of large-sized genomic DNA within an hour from several bacteria. Anal. Biochem. 278:86-90.

22.Vandecasteele, S.J., W.E. Peetermans, R. Merckx, M. Van Ranst, and J. Van Eldere. 2002. Use of gDNA as internal standard for gene expression in Staphylococci in vitro and in vivo. Biochem. Biophys. Res. Commun. 291:528-534.

Received 28 January 2003; accepted 8 April 2003.

Address correspondence to:

Tamás Henics

INTERCELL AG

Campus Vienna Biocenter 6

1030 Vienna, Austria

e-mail:thenics@intercell.com 